\title{
Abdominal Tuberculosis Presenting with an Indiscernible Abdominal Complaint: A Case Report
}

\author{
Abdulrahman A Sindi"
}

Department of Emergency Medicine, King Abdulaziz University, Jeddah, Saudi Arabia

DOI: $\underline{10.36348 / \text { sjmps.2020.v06i06.004 }}$ | Received: 01.06.2020 | Accepted: 10.06 .2020 | Published: 16.06 .2020

*Corresponding author: Abdulrahman A Sindi

\section{Abstract}

Abdominal tuberculosis accounts for $11 \%$ of patients of extra-pulmonary tuberculosis. It is also the sixth most common form of extra-pulmonary tuberculosis. Abdominal tuberculosis that presents without pulmonary or general signs/symptoms of tuberculosis is rare events that occur only in $1.2 \%$ of the disease. It can result in an intestinal obstruction; however, it is a rare cause of intestinal obstruction that is usually overlooked or misdiagnosed. We report a 17-year-old female patient who's not known to have any medical illness presented with acute abdominal pain due to small bowel obstruction with no significant effect on bowel habit. Imaging showed free fluid in the abdomen and small bowel obstruction. The patient was managed surgically with adhesiolysis and started anti-microbial therapy. The report describes the diagnostic challenges that abdominal tuberculosis can presents with, especially in patients without accompanying pulmonary or general tuberculosis signs/symptoms. Such a presentation can be a challenge to diagnosed and usually are mistaken with other differential diagnoses. Therefore, clinicians - especially in areas that are endemic with Tuberculosis - need to consider such rare diagnoses and have a high suspicion of tuberculosis in such a presentation. Keywords: Tuberculosis, abdominal tuberculosis, Intestinal obstruction, gastrointestinal tuberculosis, abdominal pain, acute abdomen.

\begin{abstract}
Copyright @ 2020: This is an open-access article distributed under the terms of the Creative Commons Attribution license which permits unrestricted use, distribution, and reproduction in any medium for non-commercial use (NonCommercial, or CC-BY-NC) provided the original author and source are credited.
\end{abstract}

\section{INTRODUCTION}

Tuberculosis was and still a significant health problem and represents one of the most common causes of death in the world [1]. Abdominal tuberculosis is a common presentation of tuberculosis that accounts for $11 \%$ of tuberculosis cases [2]. However, primary abdominal tuberculosis is not a common subset of the disease and accounts only for $1.2 \%$ of tuberculosis [3]. Abdominal pain is the presenting symptom in $88 \%$ of the abdominal tuberculosis [4]. When presenting without the history of pulmonary tuberculosis, the disease can be challenging to diagnose early, especially in non-acute settings. In addition to that, only one-third of patients with abdominal tuberculosis present with the typical systemic manifestations of tuberculosis; fever, night sweats, malaise, loss of appetite, and night sweats [5]. This presentation highlights the need for early clinical suspicion, particularly in patients presenting solely with abdominal complaints.

\section{CASE Report}

A 17-year-old previously healthy female patient presented to the emergency department (ED) with severe abdominal pain that started gradually in the past two weeks and peaked at the presentation to the ED. The pain was colicky; however, it became persistent for the last day.

The patient complained of nausea, loss of appetite, and alternating constipation with diarrhea for the past week. The patient denies fever, chills, night sweats, recent weight loss, or taking any medication. She doesn't have a history of surgical procedures, and review of system is negative.

Her vital signs are within normal limits; blood pressure is $110 / 70 \mathrm{mmHg}$, the pulse is 90 beats per minute, respiratory rate is 19 breaths per minute, oxygen saturation is $100 \%$, and her temperature is 36.7C. On examination, she is alert, oriented, and in severe pain. Her abdomen was tender, especially in the lower suprapubic area and the right lower quadrant with positive rebound tenderness. There is no guarding, 
rigidity, rashes, or scars on the abdomen. Bowel sounds are sluggish.

Her laboratory test results showed normal white blood cells $(7,620$ per microliter), negative blood pregnancy test, and otherwise normal test results. We started the patient on intravenous (IV) fluids and IV Morphine Sulfate to control the pain prior to obtaining a double-contrast Computed Tomography (CT) scan. During that time and prior to sending the patient to the radiology department, bedside ultrasound was performed to rule out appendicitis. Accidentally the patient was found to have free fluid in the pelvis around the urinary bladder and in the right iliac fossa.

The CT scan, with per os (PO) and IV contrast, was done and initially noted that the appendix could not be adequately visualized, and there was a high suspicion of a bilateral tubo-ovarian abscess. Later, the scan was reviewed/interpreted again and showed two suspicion transition points of the small bowel and an area with bowel loop dilatation suggestive of small bowel obstruction. The patient was kept nil per os (NPO) and was started on antimicrobial therapy empirically on IV Ceftazidime and Metronidazole.

The surgical team planned to perform laparotomy for the bowel obstruction. Under general anesthesia, a laparoscopic exploration was started, and upon exploration, the omentum was obstructing the view with multiple adhesions rendering difficult field visualization; therefore, the team proceeded with open laparotomy with a low midline incision. Diffuse small whitish deposits were found in the intestine, liver, and the omentum, with serosanguinous fluid encountered during the procedure See Figure-1.

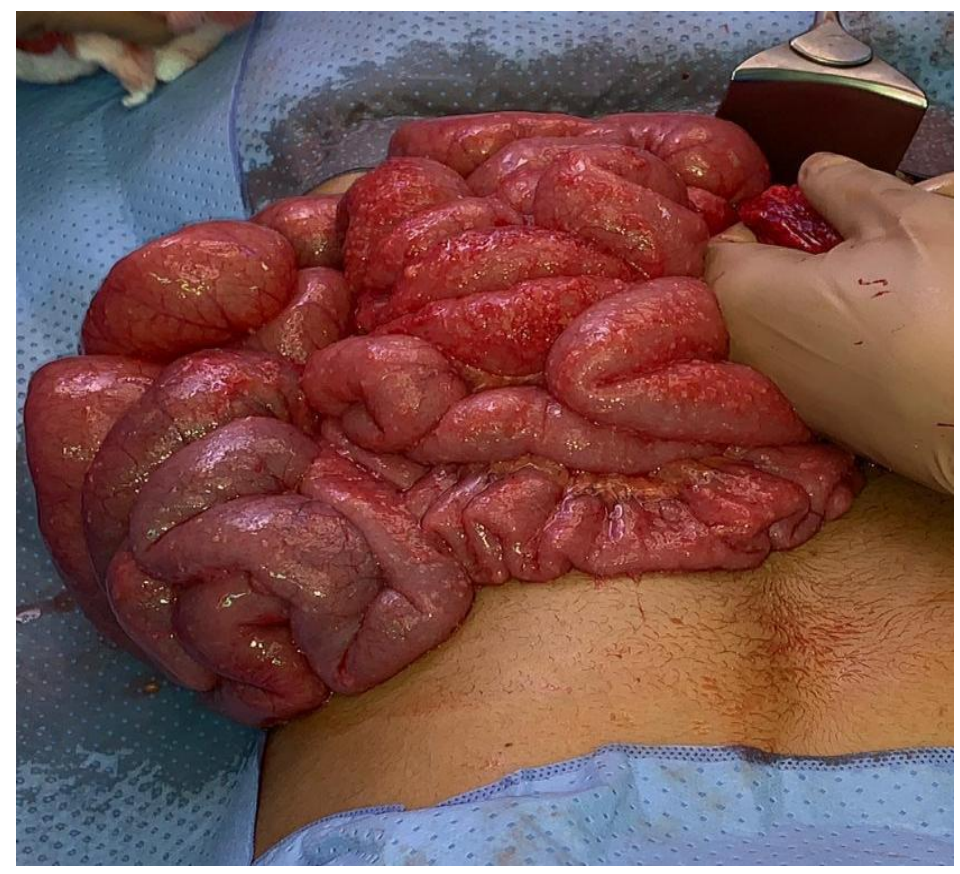

Fig-1: Intestine with many white to yellow tubercular deposites

Adhesiolysis was performed to relieve the obstruction. Excisional biopsies and fluid cytology were sent for pathological analysis. Postoperatively, the patient was vitally stable and transferred to the surgical ward.

The intraoperative specimen pathological examination showed granulomatous inflammation with focal necrosis. On bacteriological analysis of tissue specimen and ascitic fluid, the test detected trace mycobacterium tuberculosis complex. The patient was started in an anti-tuberculosis microbial regimen, including Isoniazid 300 PO once daily, Rifampicin 600 mg PO once daily, Ethambutol 1200 mg PO once daily, Pyrazinamide $1500 \mathrm{mg}$ PO once daily, Pyridoxine 40 mg PO once daily, with outpatient follow up. The patient was discharged home on day five after surgery in a stable condition.

\section{Discussion}

Abdominal tuberculosis is a major clinical disease with grave complications that can present with wide range of signs and symptoms, with abdominal pain reaching up to $88 \%$ of presentations [4]. This 17 year-old patient presented with abdominal pain in the lower part of the abdomen associated with nausea and alternating diarrhea and constipation, with no pulmonary or systemic manifestations that are often associated with tuberculosis. This presentation makes the diagnosis of abdominal tuberculosis particularly difficult to predict on initial patient encounter, favoring the more common diagnoses, such as appendicitis in these clinical settings. The usual presentation of 
abdominal tuberculosis is vague with nonspecific sign and symptoms and runs in an indolent course, with late usual presentation of the disease. Such presentation without pulmonary or general symptoms - makes it a challenging task to diagnose such case in clinical setting.

The patient here presented with acute abdomen, which makes it difficult to consider chronic/indolent diseases in her differential diagnosis. Abdominal tuberculosis can affect a single abdominal organ, mimicking appendicitis, cholecystitis [6]. When abdominal tuberculosis does so, it requires a high index of suspicion and proper clinical evaluation to avoid the complications of the disease. When abdominal tuberculosis causes bowel obstruction, early diagnosis and surgical intervention can improve the patient outcomes [7].

\section{CONCLUSION}

Abdominal tuberculosis can present with acute abdomen mimicking other more common diagnoses. Such a presentation requires clinicians to have a high index of suspicion to avoid complications and improve patient Bowel obstruction due to tuberculosis requires laparotomy and anti-tuberculous therapy.

\section{ACKNOWLEDGEMENT}

We are grateful for Dr. Emad A Fallatah for his assistance in this case report and for providing us the images from the intra-operative laparotomy.

\section{REFERENCES}

1. Patel, N., Ondhia, C., \& Ahmed, S. (2011). Bowel obstruction caused by intestinal tuberculosis: an update. Case Reports, 2011, bcr0620114361.

2. Furin, J., Cox, H., \& Pai, M. (2019). Seminar Tuberculosis.

3. Cysneiros, A. S. D., Afonso, M., Ferro, F., Carvalho, J., Mourato, T., Gomes, A., ... \& Castro, A. (2018). Retrospective study of abdominal tuberculosis without pulmonary involvement: a difficult to diagnose form of extrapulmonary tuberculosis.

4. Kumar, R. A. M. I. S. H., Saddique, M. U. H. A. M. M. A. D., Iqbal, P., \& Khan, N. A. (2007). Abdominal tuberculosis: clinical presentation and outcome. Pakistan J Surg, 23(4), 242-244.

5. Alyoune, M., Nadir, S., Merzouk, M., Mounadif, A., Biadillah, M. C., Jamil, D., ... \& Cherkaoui, A. (1994). Tuberculous anal fistulas. 13 cases. In Annales de gastroenterologie et d'hepatologie (Vol. 30, No. 1, pp. 9-11).

6. Vasan, T. S., \& Kumar, V. K. (1998). Primary tuberculous appendicitis: A Case Report. Medical journal, Armed Forces India, 54(3), 264-265.

7. Akbar, M., Haider, I. Z., Naveed, D., Akbar, I., Khattak, I., Akbar, K., \& Zafar, A. (2010). Surgical management of tuberculous small bowel obstruction. Journal of Ayub Medical College Abbottabad, 22(2), 171-175. 